\title{
Maximum-Power-Point Tracking Using Multiphase Interleaved Converters Based on Multi-Unit Synchronization
}

\author{
Niphat Jantharamin *, and Ponlawat Thongbuaban **
}

\begin{abstract}
This paper presents an application of a multiphase interleaved converter in tracking maximum power points (MPPs) of a photovoltaic (PV) panel regardless of environmental variations. Maximum power from the panel was extracted by means of the well-known the perturb-and-observe (P\&O) method. The switching control technique used an interleaving scheme based on multi-unit synchronization. The converter performed harmonic attenuation without affecting the tracking speed. This approach is straightforward, reliable and inexpensive, and could be applied to any higher number of switching cells without difficulty.
\end{abstract}

Keywords: Maximum-power-point tracking, Interleaved converter, Multi-unit synchronization

\section{Introduction}

A substantial rise in global energy demand and growing concern about shortage on conventional energy reserves as well as environmental issues have drawn more attention to PV systems. Due to weather variations, the operating point of the PV panel fluctuates and may not be at or even near the maximum power point (MPP). A major concern for such a system is to achieve high efficiency by maximum power point tracking (MPPT) for the PV panel, which is performed by a power converter. Rapid changes in voltages and currents within a switching converter cause electromagnetic interference (EMI). The converter becomes a source of interference for other equipment in its system, and its EMI also hinders its own proper operation. The most cost-effective way of dealing with EMI is to prevent the EMI from being generated at its source [1]. EMI production can be attenuated by using an interleaving technique which cancels unwanted harmonics [2]. Another advantage of using this approach is that the converter becomes more reliable and less susceptible to its own noise [1]. The circuit diagram of an $\mathrm{N}$-phase interleaved step-up converter is shown in Fig. 1. According to the interleaving technique, the identical parallel switching cells are operated at the same switching frequency with a phase shift in their switching waveforms over equal fractions of a switching

\footnotetext{
* Dept. of Electrical and Computer Engineering, Naresuan University, Thailand. (niphatj@nu.ac.th)

** Dept. of Electrical and Computer Engineering, Naresuan University, Thailand. (na_met_ta@hotmail.com)

Received 11 October 2013; Accepted 28 November 2013
}

period. Interleaving $\mathrm{N}$ cells therefore requires the cells to operate at a phase displacement of $2 \pi / \mathrm{N}$ radians with respect to one another [3]. However, as the number of switching cells of an interleaved converter is increased, the aforesaid switch control and achieving equal current sharing among the cells become more complicated [4].

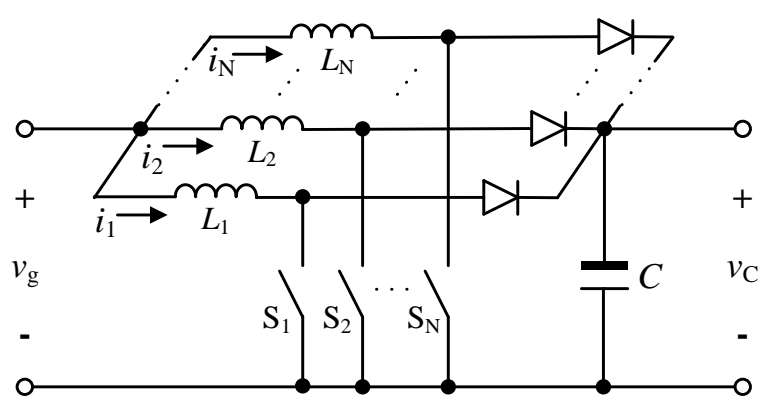

Fig. 1. An N-phase interleaved step-up converter

\section{Interleaving Technique Based on Multi-Unit Synchronization}

A straightforward, reliable, and low-cost approach of switch control for multiphase interleaved converters was implemented. This technique was devised from the concept of control-signal generation for multi-unit synchronization by which all devices were operated at the same frequency [5]. A desired switching frequency and phase shift among the parallel switching cells were dictated by a properly designed resistance-capacitance time constant for the PWM 
controllers, where trigger signals were generated by a sync pulse generator as shown in Fig. 2.

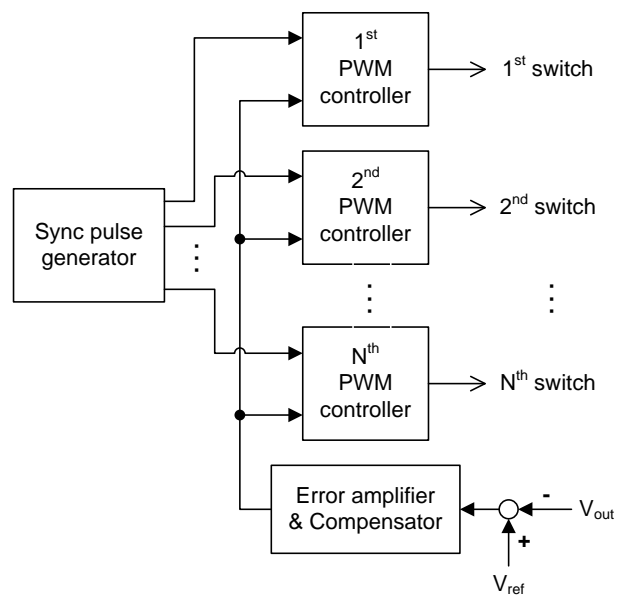

Fig. 2. Synchronization of switch-control signals

The sync pulse signals for an $\mathrm{N}$-phase interleaved converter were generated by a circuit that consisted of $\mathrm{N}$ modules of a readily available integrated circuit (IC) as shown in Fig. 3. Each module of the circuit was based on one NE555 timer IC. The number of NE555 modules was therefore dictated by the number of switching cells of the converter in question. As a consequence, the circuit performed an $\mathrm{N}$-phase sequential timer.

The procedure of sync pulse generation started with the first module producing its output signal for the first converter phase. After the output signal of the first module had ended, the second module was initiated to generate its output signal. Following the end of the output signal of the second module, the third module was started. The procedure carried on sequentially. After the last module was off, the first module then began to operate again, which referred to the beginning of the next switching period of the converter. The signal generated by each module was sent to a PWM controller for driving its corresponding converter cell, and this caused interleaving action.

The next important part of the signal generation for controlling switches of the interleaved converter was interconnection of the output signals from the NE555 modules to UC3843 ICs that drove the power switches of the converter. Each PWM controller of this technique consists of a UC3843 as shown in Fig. 4, which is a fixed frequency current-mode PWM controller. It is particularly designed for off-line and DC to DC converter applications with minimum external components. This IC includes a trimmed oscillator for exact duty cycle control, a temperature compensated reference, high gain error amplifier, current sensing comparator, and a high current totem pole output for driving a power MOSFET [6].

Normally, the timing capacitor $\left(\mathrm{C}_{\mathrm{T}}\right)$ is charged between two thresholds, namely the upper and lower voltage limits. When the $C_{T}$ initiates its charge cycle, the output of the PWM gets started and turns on. The timing capacitor is charged until it reaches the upper voltage limit of the internal comparator. Consequently, the discharge circuitry activates and discharges $\mathrm{C}_{\mathrm{T}}$ until the lower voltage limit is met. During this discharge period the PWM output is disabled, thus ensuring an off time for the output [6].

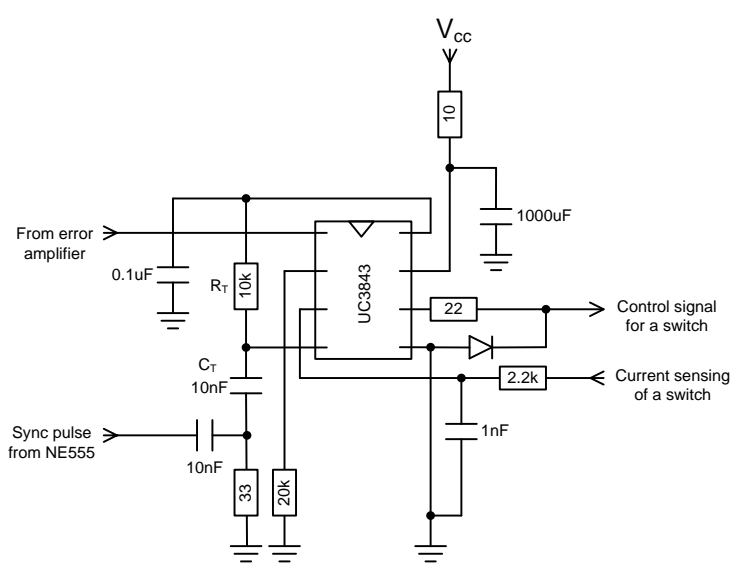

Fig. 4. PWM controller circuit for each switching cell

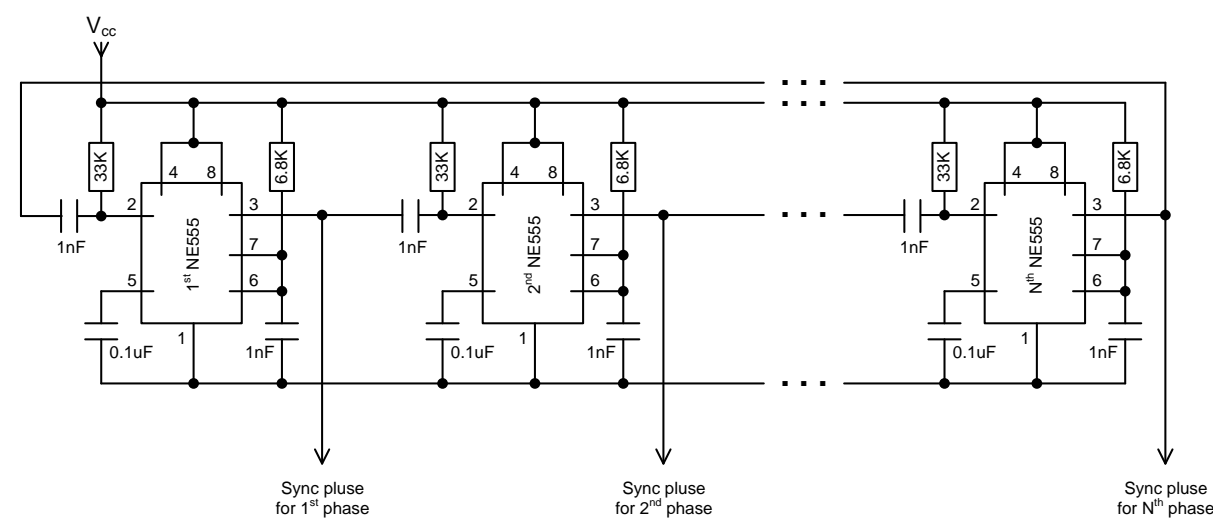

Fig. 3. Sync pulse generator circuit for an N-phase interleaved converter 
Even a slight difference in the resistance and capacitance values in the PWM controllers resulted in non-identical waveforms. However, this difference had no detrimental effect on this switch control. The synchronization was imposed in the following way. In a PWM controller (see Fig. 4), the capacitor $\mathrm{C}_{\mathrm{T}}$ was connected to ground via a $33-\Omega$ resistor. The voltage across this small resistor increased the timing-capacitor voltage above the upper voltage limit of the internal oscillator, and therefore served as a sync pulse train for its corresponding PWM controller. As a result, the controller operated at the frequency dictated by the combination of $\mathrm{R}_{\mathrm{T}}$ and $\mathrm{C}_{\mathrm{T}}$ until the sync pulses appeared. In addition, it is necessary that the UC3843 oscillator was set to a lower frequency than that of the sync pulse train. Subsequently, the modified R-C oscillator pulse train for each converter phase became input of its corresponding PWM controller. Following the R-C pulse input, the PWM controllers generated switch control signals for their switching cells with a phase shift of $2 \pi / \mathrm{N}$ radians between them [5].

\section{Maximum-Power-Point Tracking Using Multiphase Interleaved Converters}

In the literature, several methods for MPPT have been developed. Each has advantages and limitations. None of them is suitable for all applications. The decision is usually made by considering such factors as the complexity of the algorithm and circuitry, tracking accuracy, tracking speed, controller cost, available data and expertise of the user. Most of the MPPT methods have been developed for gridconnected PV systems. For a stand-alone configuration with using limited computing power, we require the MPPT scheme to not only operate in high performance, but also be simple in its implementation, hence low in computation cost. In addition, it should cause no or as little as possible disturbance to the normal system operation.

In this work, the well-known perturb-and-observe ( $\mathrm{P} \& \mathrm{O})$ method was adopted, which is most commonly used in practice because its algorithm is simple, and no prior knowledge of the PV generator is required. Here, the operating voltage of the PV panel was perturbed by adjusting the duty cycle of the interleaved converter, and the PV power output was then observed. The power value was compared with its previous value at the last sample. If the power increased, the voltage perturbation was continued in the same direction. Otherwise, if the power reduced, the direction of voltage perturbation was reversed [7]. Here, the MPPT was implemented by using a three-phase interleaved step-up converter as shown in Fig. 5. A microcontroller was used for comparison of PV power values and corresponding adjustment of the converter duty cycle.

In addition, it is necessary for an interleaved converter to ensure that each switching cell shares the load current equally in order to reduce the current stress of the switching devices and to improve the reliability of the converter. Therefore, a switch current of each cell was sensed, and the current sharing was achieved by means of a peak-current control scheme in which the peak current value of each switching cell was dictated.

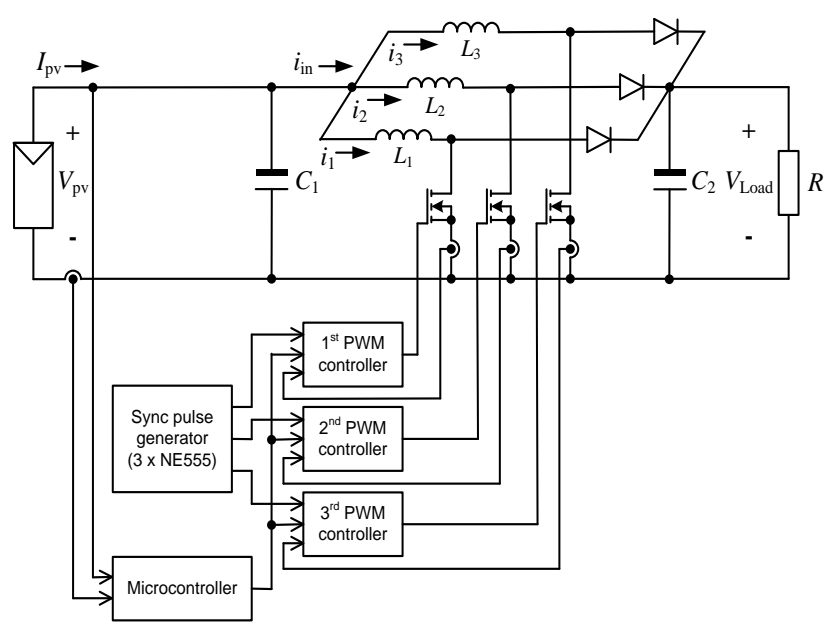

Fig. 5. Circuit diagram of the 3-phase interleaved step-up converter based on multi-unit synchronization for MPPT

\section{Experimental Results}

With an expected switching frequency of approximately $37 \mathrm{kHz}$ the switching period was about $27 \mu$ s. Therefore, the working duration of each NE555, which was equal to one-third of the switching period, was determined to be about $9 \mu \mathrm{s}$. The PWM controllers generate switch control signals for three switching cells with a phase shift of $120^{\circ}$ between them, as shown in Fig. 6. The inductor currents of the converter were always distributed equally among the cells, and their waveforms in discontinuous conduction mode (DCM) are shown in Fig. 7. Despite discontinuity of inductor current in each cell, the input current of the converter was still continuous. The current waveform of the converter input and its fast Fourier transform (FFT) are shown in Fig. 8. The experimental results showed that harmonic components of the interleaved converter terminals were remarkably attenuated.

According the MPPT scheme, the three-phase interleaved step-up converter extracted maximum power from the PV 


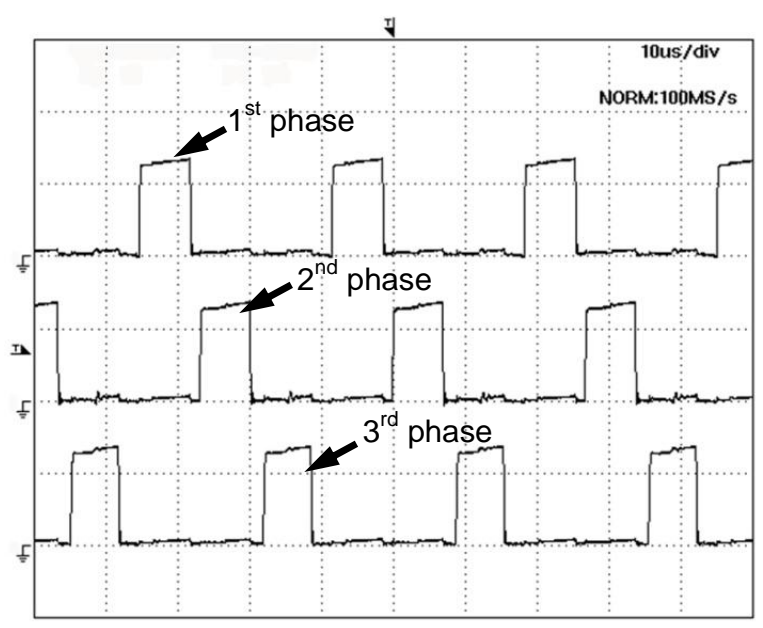

Fig. 6. Outputs of the PWM controllers serving as switch control signals for the three-phase interleaved converter

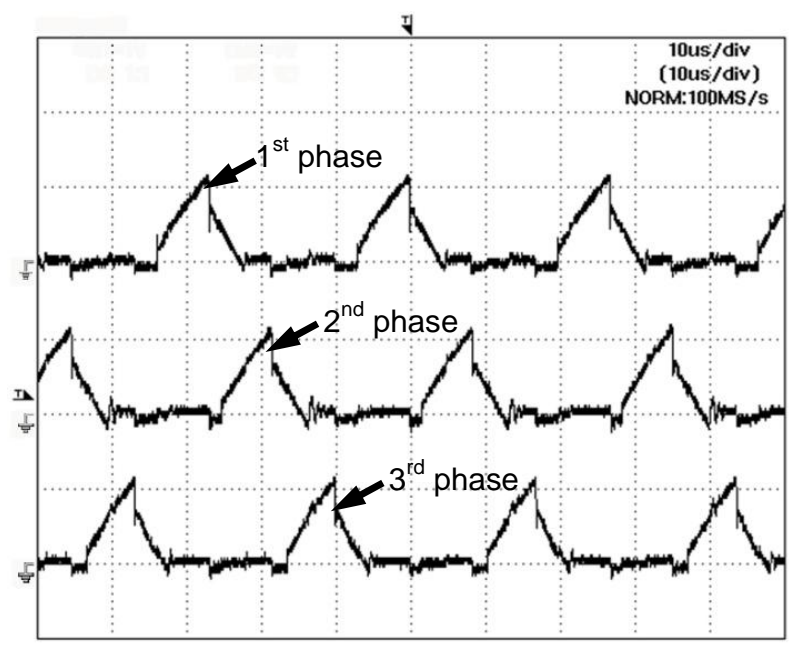

Fig. 7. Inductor current waveforms of three switching cells in DCM

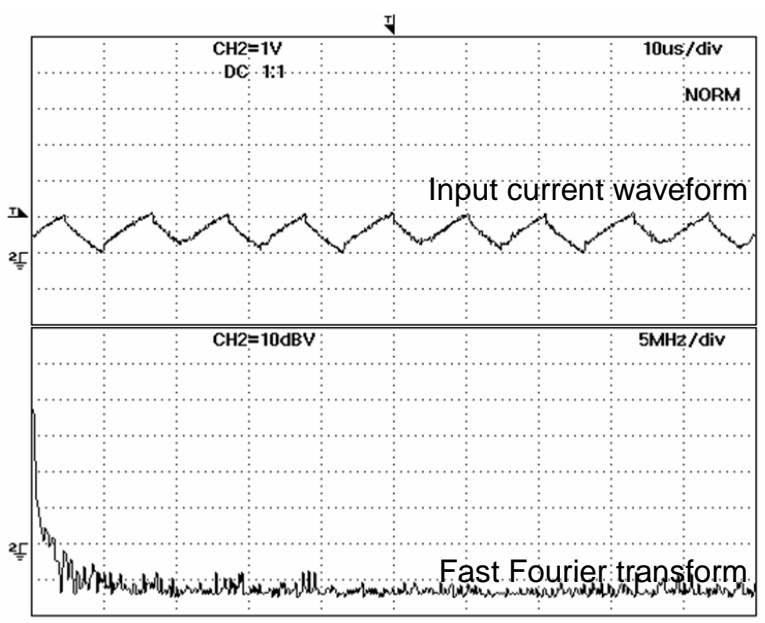

Fig. 8. Input current waveform of the interleaved converter panel under irradiance variations. As shown in Fig. 9, the MPPT test was started with the irradiance value of incident on the PV panel and the converter commenced the tracking process until the MPP was met. The PV panel then worked around the MPP, and therefore produced maximum power corresponding to the irradiance value. After the irradiance rose to $1000 \mathrm{~W} / \mathrm{m}^{2}$, the converter continued to track the MPP again resulting in an increase in the PV current and therefore the PV output power. In the event of irradiance decrease the MPPT experiment was carried out under the irradiance value of $1000 \mathrm{~W} / \mathrm{m} 2$ at the beginning as shown in Fig. 10. As expected, the MPP was tracked by the converter until the steady state of the system was reached. When the irradiance reduced to $800 \mathrm{~W} / \mathrm{m}^{2}$, the PV current dropped and so the output power of the PV panel. After the new MPP had been found, the panel gave the maximum power related to the present light intensity.

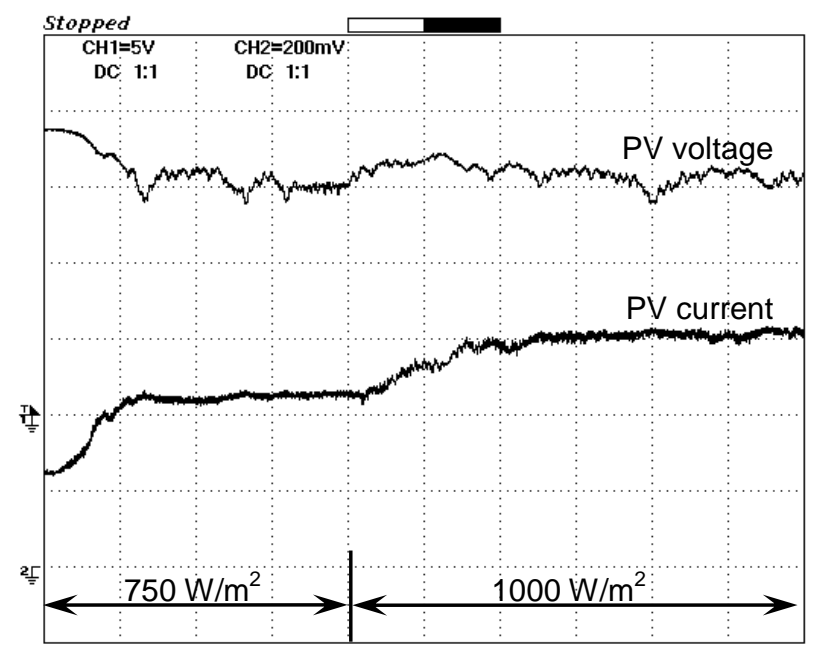

Fig. 9. MPPT during an increase of irradiance

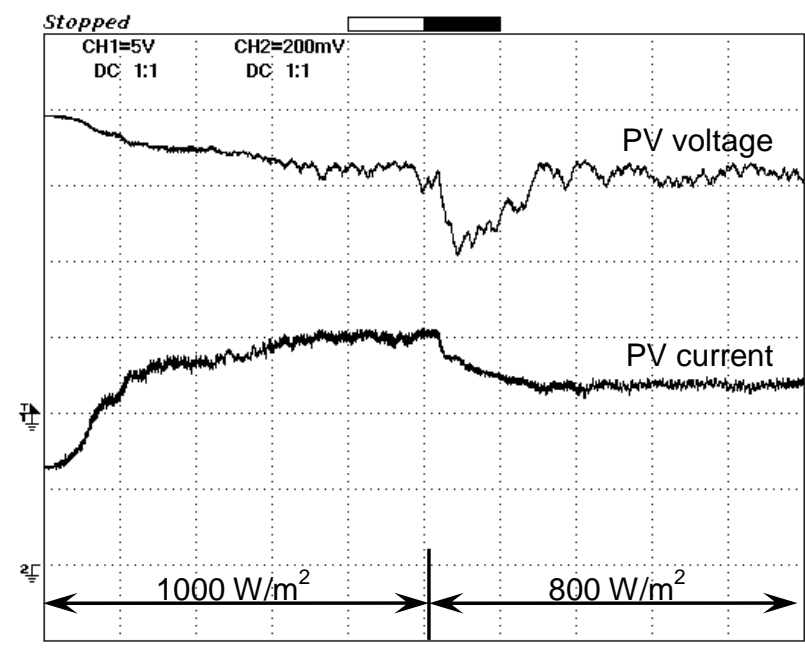

Fig. 10. MPPT during a decrease of irradiance 


\section{Conclusion}

In this paper, the application of the multiphase interleaved converter in tracking maximum power points of the PV panel was presented. The control signal generation for the converter switches was based on multi-unit synchronization approach. MPPT regardless of weather variations and current sharing among the switching cells were achieved by means of the $\mathrm{P} \& \mathrm{O}$ technique and the peak-current control. The presented switching technique was straightforward, reliable and inexpensive. In particular, this approach offered switch control that is modular - each switching cell could be implemented individually, independent of the other switching cells. Therefore, this approach could be applied to any higher number of cells without difficulty.

\section{References}

[1] N. Mohan, T. M. Undeland, and W. P. Robbins, "Power electronics: converters, applications, and design," 3rd ed: John Wiley \& Sons, 2003.

[2] P. Zumel, O. Garcia, J. A. Cobos, and J. Uceda, "EMI reduction by interleaving of power converters," in Nineteenth Annual IEEE Applied Power Electronics Conference and Exposition. APEC '04., vol. 2, 2004, pp. 688-694.

[3] B. A. Miwa, D. M. Otten, and M. E. Schlecht, "High efficiency power factor correction using interleaving techniques," in Seventh Annual IEEE Applied Power Electronics Conference and Exposition. APEC '92., 1992, pp. 557-568.

[4] J. Abu-Qahouq, M. Hong, and I. Batarseh, "Multiphase voltage-mode hysteretic controlled DC-DC converter with novel current sharing," IEEE Transactions on Power Electronics, vol. 19, no. 6, pp. 1397-1407, November 2004.

[5] P. Thongbuaban and N. Jantharamin, "New switch-control technique for multiphase interleaved converters with current sharing and voltage regulation," in the 2011 International Conference on Electrical Machines and Systems (ICEMS 2011), 2011.

[6] Texas Instruments, “Application note: UC3842/3/4/5," 1999.

[7] N. Kasa, T. Iida, and L. Chen, "Flyback inverter controlled by sensorless current MPPT for photovoltaic power system," IEEE Transactions on Industrial Electronics, vol. 52, no. 4, pp. 1145-1152, August 2005.

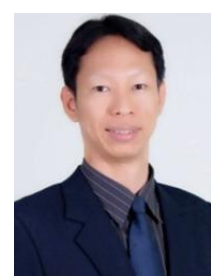

Niphat Jantharamin received B.Eng. degree from KMITL in Thailand, M.Sc. from the University of Leeds in Germany, and $\mathrm{Ph} . \mathrm{D}$. from the University of Leeds in the UK. All his degrees are in Electrical Engineering. He is a Lecturer in Electrical Engineering at Naresuan University, Thailand. His research interests are Power Electronics, Photovoltaic Systems and Efficient Energy Conversion.

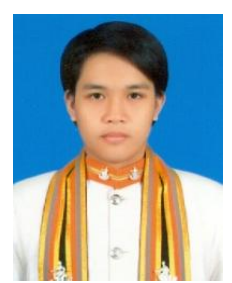

Ponlawat Thongbuaban completed his B.Eng. and M.Eng. degrees in Electrical Engineering from Naresuan University, Thailand. His research interests include Power Electronics and Photovoltaic Systems. 